\title{
CYST FORMATION IN OSTEOARTHRITIS
}

\author{
A. S. Ondrouch, Prague, Czechoslovakia \\ From the First Orthopaedic Clinic, ${ }^{*}$ Charles University, Prague
}

In the advanced stages of osteoarthritis cystic formations are frequently encountered. ranging in size from that of a pin-point to that of a cherry. They occur mainly in relation to the weight-bearing joints, and in those regions of the articular surface that are subjected to a major load and where, in advanced osteoarthritis, the cartilaginous surface has been either entirely or largely worn away.

The cysts are encountered either individually close beneath the surface bone lamella or in pairs directly opposing each other on either side of the joint line. They are usually spherical or pear-shaped and rarely of any other shape. Every cyst approaches in one point close to the articular surface; here, an opening is nearly always found, which is in most cases plugged. The walls of the cysts are formed of cancellous bone, whose trabeculae are reinforced by new bone formation. The size of the cysts is commensurate with that of the burden carried by the joint, the largest cysts being encountered in the hip joint. Connective tissue of character ranging from liquids containing fibres and cells to fibrocartilage may be found in the cysts. Debris of necrotic bone trabeculae is often found, but other tissues are rarely present. Histochemically the contents of the cysts are composed predominantly of mucopolysaccharides (Collins 1949; Harrison, Schajowicz and Trueta 1953; Françon 1956; Trueta 1957).

The sole integral theory of the origin of these cysts is that advanced by Landells (1953). In his opinion a cyst arises in the following manner: because of the uneven surface of a joint an overloading occurs at one point and causes a fracture of the terminal bone lamella. A communication is thus formed between the marrow spaces of cancellous bone and the articular cavity. In the course of the action of the joint the synovial fluid-in these cases often augmented-is then forced into the cancellous bone whose trabeculae are unable to resist such a pressure; their breakdown therefore produces a cystic cavity. After some time the stoma through which the cyst communicates with the articular cavity and which preceded the cyst proper, in some way gets stopped up. The plug is usually of newly formed connective tissue, bone or even of mere fibrin. This stoppage then terminates the process, and the cyst spreads no farther.

In the author's view this theory appeared to have several fundamental weaknesses. For instance, why should a traumatic fissure in the terminal bone lamella come about primarily, when it is known that in every healthy joint there exist physiological defects of the terminal lamella that do not lead to the formation of cysts (Ekholm and Norbäck 1951; Holmdahl and Ingelmark 1951; Ekholm 1955). It is difficult too to imagine a simultaneous breakage of the terminal lamellae at opposite points on either side of the joint occurring so often. This would be the only explanation of the frequent occurrence of paired cysts. Yet a perforation of the lamella on one side of the joint would necessarily remove the pressure at that point and remove any cause for fracture of the opposing lamella. It is doubtful too whether the synovial fluid exerts sufficient pressure or whether the fluid could be directed into the fissure. Any increase of pressure in the joint cavity would be more likely to cause distension of the capsule. It is certainly difficult to visualise the formation of some pump-like, one-way valvular system that would magnify and direct the pressure in the direction postulated.

A further difficulty is that concerning the plug of the cyst: sometimes this is soft fibrin: sometimes it is composed of material that could not be formed if it were subject to constant

$$
\text { * Principal: Professor M. Jaroš. }
$$


disturbance. It is difficult to imagine that the pressure which caused the trabeculae to break down would not damage the soft fibrin of the plug or impede the formation of connective tissue or bone.

While such considerations make it difficult to accept the whole of Landells' hypothesis, his fundamental assumption that cysts arise because of the unevenness of an articular surface

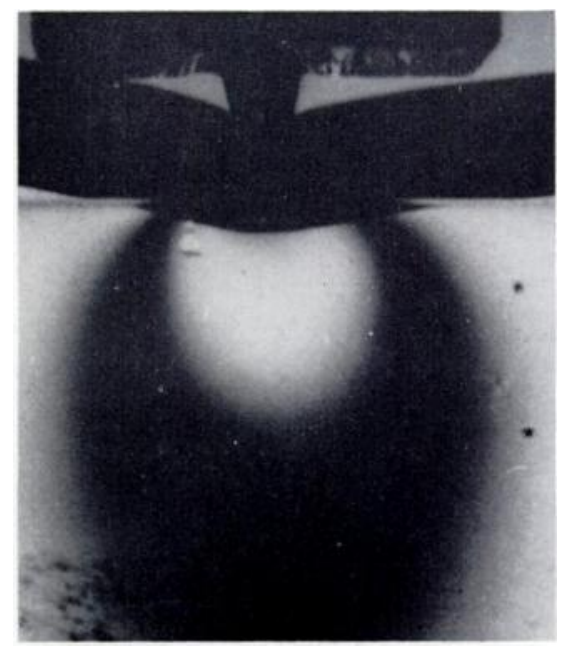

Fig. 1

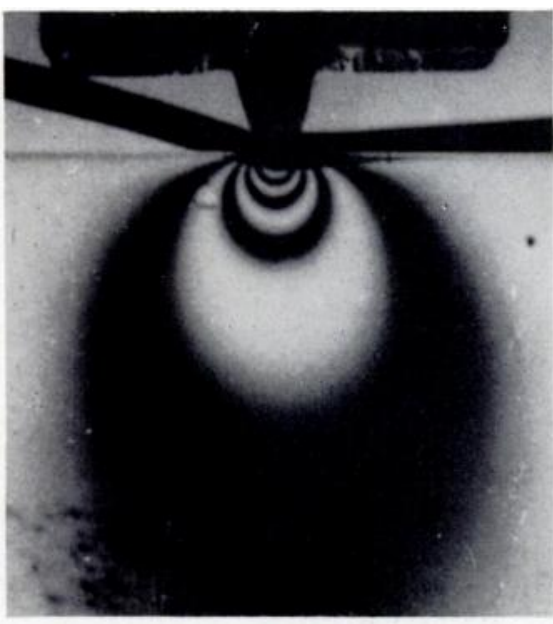

FIG. 2

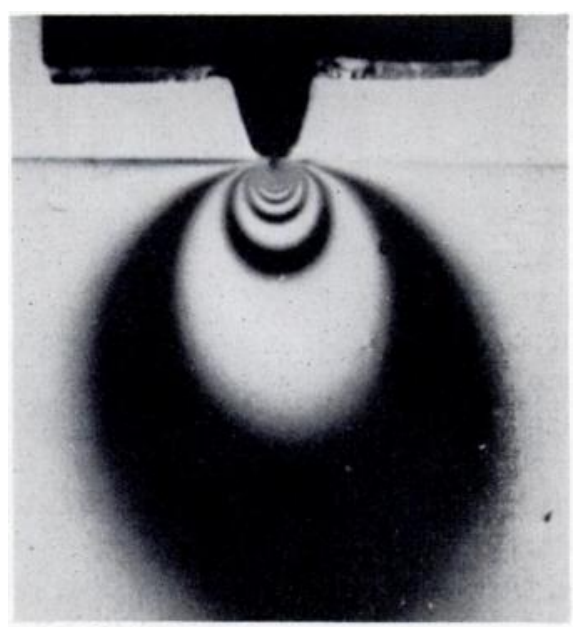

Fig. 3

Alteration of stress patterns by alteration of the area of application of load. Figure 1-Layer of foam rubber placed between the load point and the plate. Figure 2-Thin layer of hard rubber interposed. Figure 3-Metal load point pressing directly on the plate.

severely damaged by osteoarthritis cannot be challenged. It is understandable that in such a joint a concentration of load arises in those places where some point protrudes above its environment. The smaller the area of the point, the larger the concentration of stress under it. This situation is further aggravated by the absence of cartilage which normally forms a resilient pad between the surfaces of the joint, and distributes the load concentration over a wider area (Rehbein 1951). 


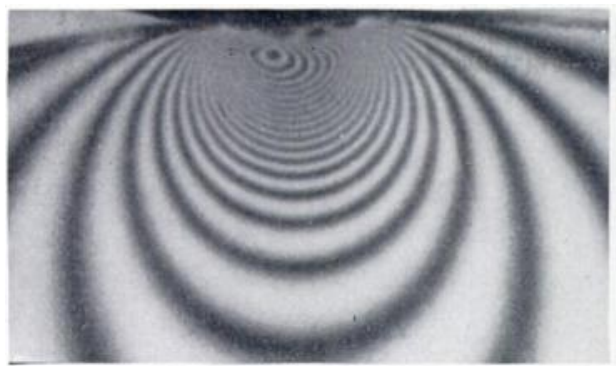

FIG. 4

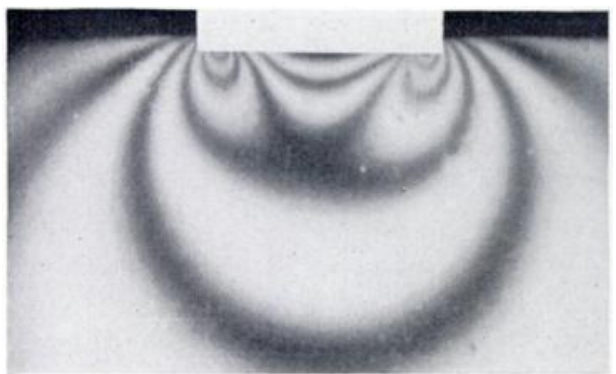

FIG. 6

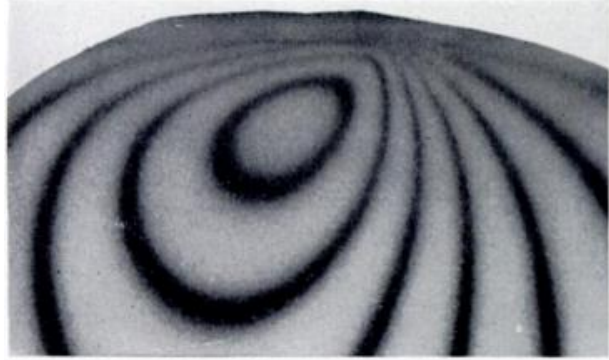

FIG. 8

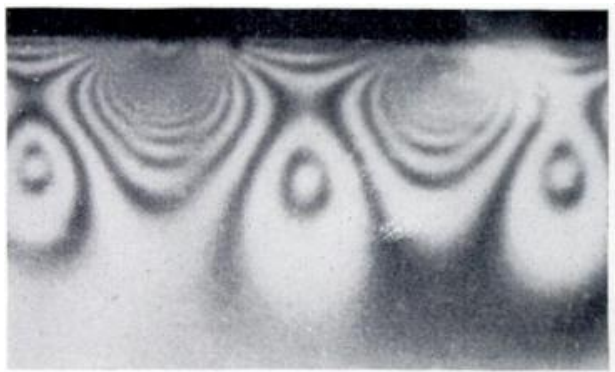

Fig. 10

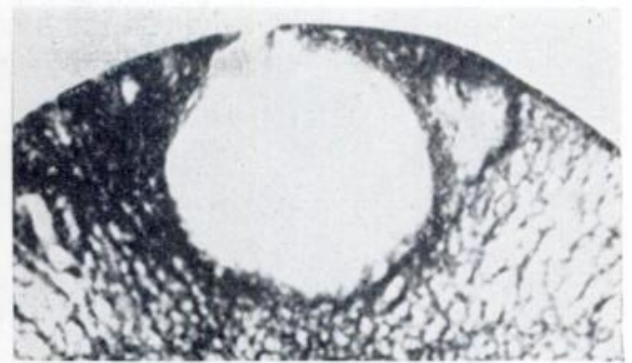

Fig. 5

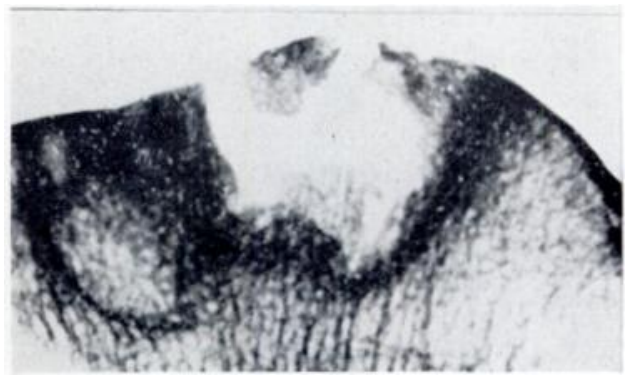

FIG. 7

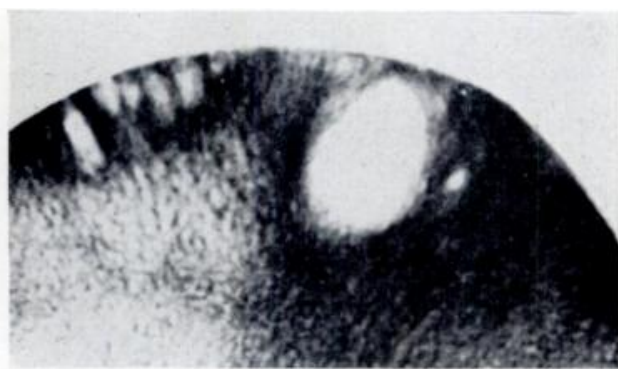

FIG. 9

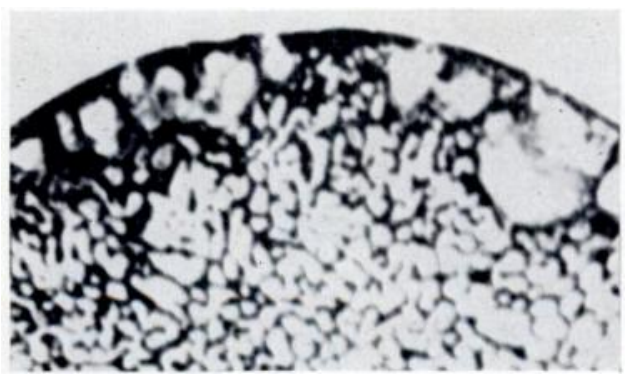

FiG. 11

Comparison of stress patterns with the shapes of actual bone cysts. Figure 4-Load point of circular shape and small area. Isochromatics of circular pattern extend far down into the model. Figure 5-A typical spherical bone cyst. Figure 6-The loading area has been increased. Concentrations of stress at the margins of the area. Figure 7-Bone cyst of shape corresponding to this pattern. Figure 8-The load has been directed obliquely at the surface of the model. Isochromatics of oval shape have been produced. Figure 9-A bone cyst of oval shape. Figure 10-Load has been applied at several points. Isochromatics with multiple concentrations have been produced. Figure 11-Multiple bone cysts of diverse shapes. 


\section{EXPERIMENTAL METHOD}

To find out more about the distribution of stress under such an overloaded protrusion of the articular surface, we carried out experimental investigations with the aid of photoelasticity models (Frocht 1946).

Photoelasticity is the property of certain materials such as organic glass of becoming temporarily refractile under load. Plane models of objects to be investigated with regard to stress distribution are made of such materials. The models are placed between crossed polaroid filters and loaded by appropriate means. Lines characteristic of a state of stress then appear in the models.

In the present investigation light circularly polarised was used to transilluminate the models and the resulting curves were termed isochromatics. These curves are an expression of the quantitative distribution of stress: that is, they are lines connecting points under equal stress. The magnitude of both main stresses becomes greater as the centre is approached.

The first series of measurements demonstrates the distribution of stress under an overloading point (Figs. 1 to 3 ). In all the three illustrations shown use was made of the same plate of optically sensitive material. The protruding irregularity of the opposite part of the joint is represented by a metal point loaded, in all three cases, with the same force. In the first case a layer of foam rubber was inserted between the point and plate (Fig. 1), in the second a thin layer of hard rubber (Fig. 2); in the third case the metal point pressed immediately against the surface of the optically sensitive plate (Fig. 3). In examination under polarised light the transparent plate shows isochromatics of an irregularly oval shape.

In the first case an isochromatic of the first order is seen (Fig. 1); in the second there is one of the fourth order (Fig. 2), and in the third there is one of up to the eighth (Fig. 3). Thus, the stress in the area immediately under the point is, in the second case, four times as large as in the first case; and in the third case, eight times as large.

The shape of the loaded (and overloaded) area varies according to the magnitude and direction of the loading force. It is interesting to compare the patterns obtained in the course of experimental loading of models with the shapes of actual cysts. For instance, in loading a circular plate of minute area we obtain isochromatics of a circular shape, with a strong concentration under the load and a perturbation that extends far downward. Corresponding to isochromatics of this shape are the spherical cysts seen in bone (Figs. 4 and 5).

By increasing the loading area the isochromatics may acquire shapes such as those shown in Figure 6. Here, apart from the region of maximum stress, concentrations appear on the edges of the loading area. Corresponding approximately to this shape is the actual cyst shown in Figure 7.

If the loading force is directed obliquely at the upper surface of the plate isochromatics of oval shape are produced (Fig. 8). This shape may be compared with that of an actual bone cyst (Fig. 9).

In the case of a simultaneous occurrence of several minor protrusions, and in the absence of a pressure damping pad, a distribution of isochromatics with multiple concentrations is produced (Fig. 10). Such local disturbances may be the cause of many cysts of diverse shapes, as shown in Figure 11.

\section{CONCLUSIONS}

As a result of these investigations, the origin of arthritic cysts appears in a substantially clearer light. The manner in which stress is distributed beneath the surface of the joint, in conditions of large loads operating on a small area, results in the formation of a specific zone of overloading at some depth below the surface. This is followed by a biological reaction to such overloading. In the region where the overloading is so large that it exceeds the limits of physiological endurance the bone dies and is liquefied. This produces a cavity shaped like the zone of overloading. Its size depends on the mechanical factors and on the biological qualities of the bone. The more resistant the tissue the smaller will be the region affected. 
Outside the area of destruction of bone there is a peripheral zone where the bone has survived, yet has felt the effects of overloading. Here the tissue is capable of a biological reaction to such overloading. This reaction consists of the apposition of new bone on to the original trabeculae.

Once the liquefaction of the central zone sets in, a fundamental change arises in the mechanical relations of the loaded region. The liquid content of the cysts is unable to withstand the load, which is therefore transferred to and fully concentrated on the thin layer separating the cyst from the articular cavity. In most cases this layer breaks down and may be pushed into the cyst. A stoma is formed through which the cysts communicate with the articular cavity.

The entire process may be represented by a model (Figs. 12 to 14). The plate is loaded, the point of the loading element representing the uneven surface of the bone (Fig. 12). The cyst under force may be demonstrated by a drilled hole filled up with soft rubber without great bearing capacity. The concentration of stress is then transferred to the bridge (Fig. 13). By an increase of load or, as will probably be the actual event, by a sudden impact, the bridge is fractured. The breach in the roof of the cyst ends the stress concentration in this region, for the prominence that had caused it has now disappeared. Its pressing in brings another point or points of the articular surface into relative prominence, and these in turn become the foci for the formation of further cysts (Fig. 14).

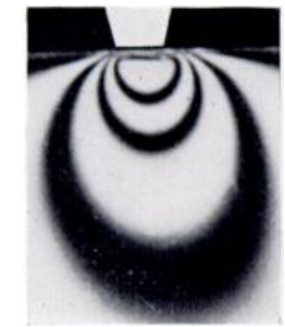

Fig. 12

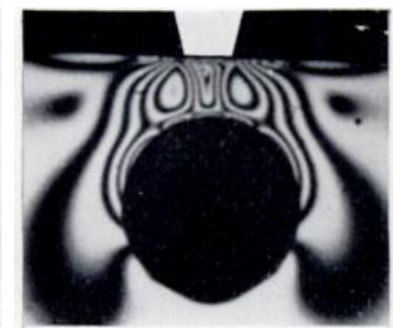

FIG. 13

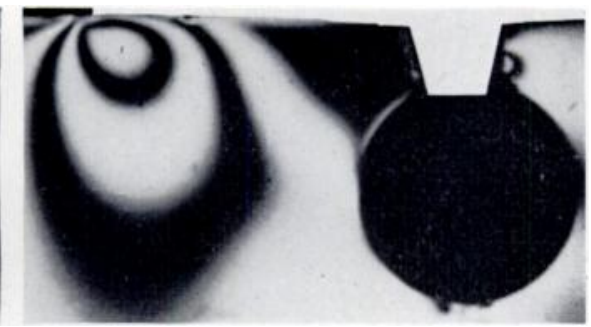

FIG. 14

The sequence of events leading to fracture of the roof of the cyst. Figure 12-Load producing isochromatics in the model. Figure 13-The cyst is represented in the model by a hole filled with soft rubber. The concentration of stress is now transferred to the "bridge "-in other words the roof of the cyst. Figure 14-Fracture of the bridge ends the concentration of stress in this region, and another point comes under load.

At times the cystic destruction of a joint may be so rapid that cysts are formed in between those already created. This entails a substantial change of terrain. Because of the condensed bone tissue in the vicinity of the older cysts, the new cyst is formed in a region where the character of the tissue is extremely variable. It is thus possible to explain those prolonged, well-nigh cylindrical shapes of minute multiple cysts. Where two adjacent cysts are formed simultaneously an interaction of stress effects takes place resulting, in the end, in a coalescence of the cystic cavities. In this way heart-shaped and wedge-shaped cysts come into being (Fig. 6).

Identical changes can take place simultaneously on both sides of the joint, since on either side an identical cause is operative. We can then easily explain the origin of the "kissing" cysts of a common hour-glass shape. On the contrary, it is rather astonishing that this phenomenon does not occur every time. For instance, cysts tend to form rather in the head of the femur than in the acetabulum. This may be because the texture of the acetabular bone is more resistant.

\section{SUMMARY}

1. The cause of cyst formation lies in the major overloading of certain regions caused by the unevenness of the articular surface produced by the arthritic process.

VOl. 45 B, NO. 4, NOVEMBER 1963 
2. Photoelasticity investigations disclose an overburdening of the bone beneath the surface of the joint, which in turn markedly resembles the shapes of actual cysts.

3. The results of this investigation fit organically into the logical sequence of processes in accordance with our theory.

4. This theory is further borne out by the successful formation of cyst phenomena in healthy joints by overloading (Trias 1961).

5. Our theory has no immediate practical bearing on the treatment of arthritic cysts. However, it uncovers certain interesting aspects of the behaviour of bone tissues under mechanical overloading above the limits of biological, though below those of mechanical bearing capacity. Conclusions arising therefrom might in many respects be useful in research into the physiology of bone.

\section{REFERENCES}

Collins, D. H. (1949): The Pathology of Articular and Spinal Diseases. London: Edward Arnold \& Company. Екноцм, R. (1955): Nutrition of Articular Cartilage. Acta Anatomica, 24, 329.

Екноцм, R., and Norв̈̈ск, B. (1951): On the Relationship Between Articular Changes and Function. Acta Orthopaedica Scandinavica, 21, 81-98.

Françon, F. (1956): Coxarthrose. Basel: Documenta Rheumatologica Geigy.

Frocht, M. M. (1946): Photoclasticity, I, II. New York: John Wiley \& Sons Inc. London: Chapman \& Hall Ltd.

Harrison, H. M., Schajowicz, F., and Trueta, J. (1953): Osteoarthritis of the Hip: A Study of the Nature and Evolution of the Disease. Journal of Bone and Joint Surgery, 35-B, 598.

Holmdahl, D. E., and Ingelmark, B. E. (1951): The Contact Between the Articular Cartilage and the Medullary Cavities of the Bones. Acta Anatomica, 12, 341.

LANDells, J. W. (1953): The Bone Cysts of Osteoarthritis. Journal of Bone and Joint Surgery, 35-B, 643.

RehBein, F. (1951): Rhythmische Dauerbeanspruchung spongiösen Knochens. Jena: Gustav Fischer.

Trias, A. (1961): Effect of Persistent Pressure on the Articular Cartilage. Journal of Bone and Joint Surgery, 43-B, 376.

Trueta, J. (1957): Consideration on the Pathology of Osteoarthritis of the Hip. Septième Congrès de la Société Internationale de Chirurgie Orthopédique, p. 857. Bruxelles: Imprimerie des Sciences. 